\title{
APLICAÇÃO DO FRAM PARA SOLUÇÃO DE PROBLEMAS EM SISTEMAS SÓCIO-TÉCNICOS COMPLEXOS: ESTUDO DE CASO EM UMA UNIDADE HOSPITALAR
}

\author{
APPLYING FRAM FOR PROBLEM SOLVING IN COMPLEX SOCIO- \\ TECHINCAL SYSTEMS: CASE STUDY OF A HOSPITAL UNIT
}

\author{
Fábio Rodrigues Priori^ E-mail: fabiopriori@gmail.com \\ Tarcisio Abreu Saurin* E-mail: saurin@ufrgs.br \\ *Universidade Federal do Rio Grande do Sul (UFRGS), Porto Alegre, RS
}

\begin{abstract}
Resumo: Caracterizado pelo ambiente em constante mudança, o setor hospitalar possui problemas complexos, cuja solução necessita de uma abordagem adequada e robusta. $O$ Functional Resonance Analysis Method (FRAM), método desenvolvido para modelagem de sistemas complexos, demonstra potencial como alternativa para solução de problemas no ambiente hospitalar. Por tratar-se de um método relativamente novo, a proposta desse estudo é analisar o desempenho do FRAM quando utilizado para a solução de problemas em uma unidade de internação de um hospital universitário. Para esse fim, as ações de melhoria propostas como resultado da aplicação do FRAM foram avaliadas de acordo com os critérios de dificuldade de implantação, a possibilidade de replicação, o tempo necessário para aplicação do método, o número de envolvidos, a necessidade de capacitação e o número total de oportunidade de melhoria identificadas. No contexto avaliado, FRAM resultou em ações de melhoria consideradas satisfatórias pelos envolvidos na aplicação, destacando-se pela visão realista que proporcionou aos participantes quanto à forma que o trabalho é rotineiramente executado.
\end{abstract}

Palavras-chave: FRAM. Solução de problemas. Hospital. Erros de medicação. Prescrição.

Abstract: Characterized by its constant changing nature, healthcare sector has complex problems, which needs a proper and robust approach. The Functional Resonance Analysis Method (FRAM), a method developed for modeling complex systems, shows potential as an alternative to problem solving in the hospital environment. Since it is a relatively new method, this study proposes to analyze FRAM performance when used for problem solving in an inpatient unit of an academic hospital. For this, the results of FRAM utilization were evaluated according to the following criteria: the total number of improvement actions proposed, implementation difficult, replication possibility along the departments of the hospital, the time required to apply the method, the number of people involved and the need of training for the team. In the evaluated context, FRAM application resulted in improvement actions that were considered satisfactory by the involved team, providing a realistic view of how the work is routinely done.

Keywords: FRAM. Problem solving. Hospital. Medication erros. Prescription.

\section{INTRODUÇÃO}

Parte fundamental da melhoria contínua, os métodos de solução de problemas e investigação de acidentes costumam se restringir à avaliação de eventos passados, delegando a análise de potenciais eventos futuros para 
ferramentas de análise de riscos. Todavia, a variabilidade presente nos problemas e acidentes também está presente no trabalho normal do dia-a-dia (Hollnagel, 2012). Os modelos causais de acidentes são parte fundamental dos métodos de investigação desses eventos, sendo diferentes modelos utilizados por diferentes métodos. Leveson (2001) explica que os modelos de acidentes são utilizados com três propósitos distintos: 1) entender e investigar acidentes que ocorreram; 2) prevenir futuros acidentes; e 3) analisar o risco associado a uma atividade, ao uso de um produto ou a operação de um sistema. Leveson (2001) acrescenta que um mesmo modelo pode não ser o mais apropriado para cada um dos três propósitos.

Hollnagel (2012) afirma que investigações de acidentes são focadas em encontrar o que saiu errado, o que pode ser válido para sistemas técnicos. Contudo, segundo o autor, essa é uma visão limitada quando sistemas sóciotécnicos complexos (SSC) são o foco. Isso ocorre pois é impossível prever com precisão o desempenho de um SSC, considerando que o trabalho real não é completamente correspondente ao trabalho como imaginado (Hollnagel et al., 2014). Marais et al. (2004) reforçam a necessidade de uma nova abordagem para a segurança em SSC, defendendo que a segurança nesses sistemas deve ser tratada como uma propriedade do sistema como um todo, e não como uma propriedade de seus componentes, o que ocorre nos métodos tradicionais de investigação de acidentes.

A variabilidade do processo é causada por diversas fontes que podem ou não ser controladas, podendo incorrer tanto em resultados positivos quanto negativos. Isso porque um SSC é constituído de diversos subsistemas, os quais apesar de possivelmente possuírem variabilidade dentro dos limites do processo, em conjunto com os demais subsistemas geram o fenômeno da ressonância funcional, resultando em variabilidade além da tolerada (Hollnagel e Goteman, 2004). Enquanto as metodologias tradicionais objetivam reduzir a variabilidade de processos para aumentar a frequência das conformidades, essa abordagem só pode ser realizada em subsistemas individuais, possuindo menor impacto nas variabilidades que geram a ressonância (Hollnagel, 2012). Dessa premissa surgiu o FRAM (Functional Resonance Analysis Method), metodologia que busca adequar os processos à variabilidade inerente a eles, abordando todo o sistema sócio-técnico, de forma a reduzir a ressonância funcional. O FRAM 
parte do princípio que resultados positivos e negativos de um processo têm a mesma origem e mecanismos. Por isso, é importante partir a análise do funcionamento normal do sistema, e não de um cenário de falha (Hollnagel, 2012).

Modelos de análise de acidentes tradicionais, desenvolvidos em diferentes setores, não são diretamente transferíveis para SSC como o setor da saúde, que se caracteriza por suas interações complexas e dificuldade de padronização (Magrabi et al., 2007). Braithwaite et al. (2009) corroboram que o setor da saúde apresenta características correspondentes a SSC, concluindo que abordagens tradicionais para solução de problemas são inadequadas no contexto apresentado. Dessa forma, o FRAM, cujo modelo é baseado em funções do sistema ao invés de componentes ou estruturas, demonstra ser adequado para aplicação em SSC como o setor da saúde, permitindo uma análise não linear dos eventos (Hollnagel, 2008).

Esse trabalho objetiva realizar uma aplicação do FRAM em um ambiente hospitalar, avaliando o desempenho do método nesse contexto. O FRAM será aplicado no processo de prescrição e administração de medicamentos, em um hospital universitário.

\section{REFERENCIAL TEÓRICO}

\subsection{FRAM}

Diante da demanda por um método que abrangesse os conceitos mais modernos da engenharia de resiliência e permitisse abordar segurança mesmo em sistemas sócio-técnicos complexos, foi desenvolvido o FRAM (Functional Resonance Analysis Method), método que pode ser utilizado tanto para investigação de acidentes complexos quanto para identificar riscos em sistemas dinâmicos (Hollnagel e Goteman, 2004). Segundo Hollnagel (2012) o FRAM foi desenvolvido com o propósito específico de proporcionar uma metodologia que focasse na natureza das atividades rotineiras, ao invés de na natureza das falhas. De forma a garantir que o método atingiria seus objetivos, quatro princípios foram adotados como base para sua construção. 
O primeiro princípio corresponde a um dos preceitos da engenharia de resiliência, a premissa de que falhas e sucessos são equivalentes no sentido de que têm a mesma origem, ou seja: os motivos do funcionamento adequado ou inadequado de um sistema são os mesmos. "Fracassos e sucessos são equivalentes no sentido de que apenas é possível definir se uma ação precedente foi correta ou errada após conhecer o resultado" (Hollnagel, 2012).

O segundo princípio parte da premissa de que as pessoas sempre ajustam suas ações para adequá-las ao ambiente que se encontram. Hollnagel et al. (2014) afirmam que as condições reais de trabalho nunca correspondem ao esperado ou desejado, tornando necessária a constante adaptação do desempenho para se adequar às condições existentes, em todos níveis organizacionais. Pelo fato dos recursos disponíveis normalmente serem limitados, as adaptações feitas são mais aproximadas do que precisas, causando uma variabilidade que na maioria dos casos é suficiente para o processo desempenhar de maneira adequada, mas que também é o motivo de erros (Hollnagel et al., 2014).

O terceiro princípio dispõe que muitos dos resultados que são percebidos, bem como muitos que não percebidos, devem ser descritos como emergentes ao invés de resultantes (Hollnagel, 2012). A variabilidade resultante de ajustes cotidianos, sozinha, raramente é suficientemente significativa para causar um erro, ou grande suficiente para ser descrita como uma falha (Hollnagel et al., 2014). Quando é impossível encontrar uma causa específica que explique consequências observadas, não se pode afirmar que os resultados foram gerados por certa condição ou situação. A variabilidade gerada pelos ajustes cotidianos não é detectada, dessa forma tanto erros quanto o trabalho cotidiano podem ser descritos como emergentes da variabilidade, e não resultantes dela, ou causados por ela (Hollnagel et al., 2014).

O último princípio trata da ressonância; Hollnagel et al. (2014) observam que a variabilidade de algumas funções podem influenciar mutuamente umas às outras, aumentando a variabilidade de forma que pode afetar outras funções, num fenômeno análogo à ressonância. A ressonância impede uma análise linear, pois não é possível identificar nesse fenômeno causas específicas. Hollnagel (2012) salienta que a ressonância é utilizada no FRAM como uma 
analogia, visto que ao contrário das ressonâncias normal e estocástica, a ressonância funcional não pode ser expressa matematicamente.

Uma investigação baseada no FRAM não inicia com a busca por uma causa, mas tentando-se entender o que deveria ter acontecido em uma situação rotineira, e então se utilizando desse entendimento para explicar porque isso não aconteceu (Hollnagel, 2012). Hollnagel (2012) apresenta quatro passos para execução do FRAM, adicionando que um passo preliminar à aplicação do método é definir se a análise é relativa à investigação de um evento que já ocorreu, ou a uma avaliação de risco focada em algo que pode ocorrer no futuro. A base tanto de uma investigação de acidente quanto de uma análise de riscos utilizando o FRAM é delinear as funções fundamentais para dado cenário ou atividade (Hollnagel e Goteman, 2004).

O primeiro passo consiste em identificar as funções necessárias para execução do trabalho rotineiro. Hollnagel et al. (2014) afirmam que a melhor fonte de informações sobre as atividades de interesse são as pessoas que realmente as executam. Uma função é definida através de seis aspectos, descritos a seguir (Hollnagel e Goteman, 2004; Sawaragi et al., 2006; Hollnagel, 2012; Hollnagel et al., 2014):

- Entradas (I) - requisitos para a ativação da função. Entradas constituem em ligações com outras funções que serão utilizadas para a produção das saídas.

- Saídas (O) - Saída é o produto de uma função. A saída de uma função pode constituir a entrada de uma função subsequente.

- Recursos (R) - Recursos são aspectos necessários para função processar a Entrada.

- Controles (C) - Controles servem para supervisionar, ou restringir, a função, de forma que ela seja ajustada em caso de desvios. Controles podem tanto ser funções ativas quanto procedimentos, planos e orientações.

- Pré-condições $(P)$ - condições prévias necessárias, mas não suficientes, para ativação da função. São etapas ou condições que devem ser atendidas antes que a função possa processar a entrada. 
- Tempo (T) - Tempo é um recurso específico, presente em todo tipo de processo. Pode atuar como uma restrição, caso haja uma janela de tempo que deve ser atendida para operação da função.

O segundo passo na aplicação é a caracterização da variabilidade das funções que constituem o modelo FRAM, ponto de partida para compreensão de como as interconexões das funções podem levar a resultados inesperados. Essa etapa deve abordar tanto a variabilidade potencial, referente ao modelo, quanto a variabilidade esperada, referente à instanciação do modelo (Hollnagel, 2012). A análise é mais focada na variabilidade da saída do que na variabilidade das funções em si, pois existem outras fontes de variabilidade que podem afetar a saída, além da função (Hollnagel et al., 2014). Hollnagel et al. (2014) sugerem iniciar a descrição da variabilidade da saída das funções de forma simplificada, em termos de tempo e precisão. Em relação ao tempo, a saída pode ocorrer muito cedo, na hora, muito tarde ou não ocorrer. Em termos de precisão, a saída pode ser precisa, aceitável ou imprecisa.

O terceiro passo consiste na agregação da variabilidade real ao modelo. O modelo FRAM não representa uma situação específica, ele representa apenas a variabilidade potencial do desempenho, dessa forma nessa etapa realizam-se instanciações do modelo em determinadas circunstâncias e condições, buscando identificar como a variabilidade potencial pode se tornar variabilidade real. Essa análise não se limita à variabilidade interna das funções, devendo incluir a combinação da variabilidade nas interações, buscando como pode surgir a ressonância funcional (Hollnagel, 2012). Lundblad et al. (2008) observam que essa etapa da aplicação do FRAM é fortemente dependente da visualização do modelo, o que pode ser confuso em sistemas muito grandes ou complexos; para amenizar essa dificuldade, os autores sugerem algumas alterações estéticas no modelo, como o uso de setas para indicar a direção dos acoplamentos, no intuito de facilitar a visualização.

O quarto, e último, passo da aplicação do FRAM consiste em propor maneiras de controlar consequências de variabilidade não controlada. Nessa etapa, deve-se ter em mente que a variabilidade, mesmo fora de controle, também pode induzir a resultados positivos, nesses casos o propósito é facilitar esses resultados sem implicar na perda de controle (Hollnagel, 2012). Formas de detectar a variabilidade, como indicadores, por exemplo, devem ser adotadas 
para permitir o controle (Hollnagel et al., 2014). Lundblad et al. (2008) verificam que a etapa de seleção de contramedidas no FRAM carece de um procedimento mais estruturado, indicando que uma melhor orientação para execução dessa etapa será uma evolução para o método.

Apesar de ser um método relativamente recente, encontra-se na literatura uma diversidade de aplicações do FRAM em diferentes setores e cenários, as quais demonstram que o método FRAM pode ser utilizado para predizer ocorrências e possíveis dificuldades que emergem de interações complexas entre humanos, máquinas e fatores organizacionais (Sawaragi et al., 2006; Lundblad et al., 2008; Sujan e Felici, 2012; Rosa et al., 2014; Patriarca et al., 2017). Herrera e Woltjer (2010) acrescentam que o FRAM proporciona um melhor entendimento da relação das falhas com a rotina de trabalho, justamente por focar na variabilidade cotidiana do sistema, e não apenas nas fraquezas dele, possibilitando recomendações mais assertivas para redução dos riscos. Hollnagel (2008) indica a aplicação do método FRAM em sistemas que classifica como intratáveis e firmemente acoplados, devida à alta complexidade sóciotécnica envolvida. Belmonte et al. (2011) concluem que o FRAM é complementar a métodos clássicos de análise, considerando o uso combinado desses um passo adiante para melhor compreensão das atividades como realmente são executadas, levando em consideração fatores psicológicos e ergonômicos. Os autores acrescentam que uma melhoria proveitosa para o FRAM seria a inclusão de aspectos quantitativos, permitindo estimar a probabilidade de ocorrência dos diferentes cenários usando o modelo funcional.

\subsection{Erros de Medicação}

Bates et al. (1995) consideram erro de medicação qualquer erro que ocorra em qualquer etapa entre o processo da prescrição até a administração do medicamento, concluindo que erros de medicação são comuns, e que os erros mais graves são os relacionados a erros provenientes da prescrição médica. Teixeira e Cassiani (2010) corroboram que erros de prescrição são ocorrências comuns, sugerindo o uso da prescrição eletrônica como uma estratégia para combater esses erros. Cassiani et al. (2003) analisam a eficiência da utilização da prescrição eletrônica para redução de erros de prescrição, concluindo que o 
sistema computadorizado, apesar de não erradicar a possibilidade de ocorrência de erros na medicação, representa um avanço no sentido de minimizar erros decorrentes de prescrições mal formuladas.

Bates (2000) ressalta o desempenho positivo do uso de tecnologia no ambiente hospitalar, destacando a prescrição eletrônica que, segundo o autor, é responsável pelo maior impacto na redução dos erros de medicação, mas salientando que para o uso da automação atingir o potencial de aumentar a segurança em todas as etapas do processo, deve ser cumprida a condição de que todas as etapas estejam eletronicamente interligadas, permitindo assim a dedicação dos profissionais para atividades mais recompensadoras. Para integrar a automatização na cadeia de medicação, a etapa seguinte à prescrição eletrônica que deve ser controlada eletronicamente é a dispensa de medicamentos. Shojania et al. (2001) apresentam uma análise dos modelos de dispensários eletrônicos para medicamentos mais utilizados, verificando que, apesar de reduzir o tempo necessário para a administração dos medicamentos e melhorar a eficiência dos registros, a utilização de dispensários eletrônicos não incorre em redução uniforme de erros de medicação.

\subsection{Aplicações do FRAM}

A literatura apresenta diversas aplicações do FRAM em diferentes contextos do setor da saúde. Ao aplicar o FRAM em um ambiente de emergência hospitalar, Sujan e Felici (2012) relatam que o método exige a consideração de aspectos relacionados ao contexto que normalmente não são incluídos em modelos sequenciais; além de permitir mais liberdade de especulação sobre possíveis fontes de variabilidade, devido à natureza mais qualitativa do método. Alm e Woltjer (2010) utilizam o FRAM para investigação de um acidente ocorrido durante um procedimento cirúrgico, através da qual os autores concluem que o método é aplicável para investigações no setor da saúde, observando que permitiu um entendimento mais profundo da complexa situação analisada. Hounsgaard (2016) acrescenta que FRAM explica que um incidente de risco ao paciente é causado pela variabilidade do desempenho do trabalho cotidiano, concluindo que o método ajuda os profissionais da saúde a perceberem que mesmo pequenos ajustes no trabalho rotineiro podem levar a um impacto 
indesejado e inesperado para o paciente. Saurin et al. (2015) apresentam uma comparação da aplicação do FRAM com práticas Lean em um ambiente hospitalar, através da qual os autores verificam que o método proporciona informações sobre porque o trabalho como é feito não corresponde ao trabalho como é imaginado, além de identificar variabilidades no sistema, cujo impacto não necessariamente ocorre de forma linear ao longo do processo, sendo complementar ao Lean em vários aspectos.

As divergências entre o trabalho como é feito e como é imaginado são aprofundadas por Pickup et al. (2018), que apresentam um estudo de caso no qual o FRAM foi utilizado para identificar o impacto da variabilidade em um processo de coleta de amostras de sangue em pacientes, concluindo que o FRAM proporcionou melhor entendimento de como o trabalho é realmente executado e das origens da variabilidade em um ambiente complexo. Raben et al. (2018), em uma abordagem mais preventiva, propõe um método para identificar indicadores chave no setor da saúde, utilizando-se, para tanto, do FRAM para compreender e mapear um processo complexo de um hospital dinamarquês. No referido estudo o FRAM foi considerado uma ferramenta adequada para estabelecer indicadores, pois, segundo os autores, o método auxiliou na identificação de fontes de variabilidade, conexões do processo e resultados da variabilidade, assim, possibilitando o estabelecimento de indicadores para funções cuja variabilidade afetaria o resultado do processo. Avaliando o potencial do FRAM como ferramenta de melhoria de processos, Ross et al. (2018) aplicam o método para identificar oportunidades de melhoria em um procedimento odontológico. Para este fim, os autores buscaram identificar quais funções que, se combinadas, poderiam gerar uma ressonância positiva na saída do processo, avaliando diferentes intervenções possíveis através de instanciações do modelo FRAM.

\section{MÉTODO DE PESQUISA}

\subsection{Estratégia e delineamento da pesquisa}

A estratégia de pesquisa adotada nesse trabalho foi o estudo de caso. Assim, foi selecionado um caso relevante e a coleta de dados usou múltiplas 
fontes de evidência. O estudo foi realizado em um hospital universitário de grande porte localizado na região sul do Brasil. Integrante da rede de hospitais universitários do Ministério da Educação (MEC) e vinculado academicamente a uma universidade federal, o hospital em questão tem a missão de oferecer serviços assistenciais à comunidade, além de ser área de ensino para a universidade e promover a realização de pesquisas científicas e tecnológicas. $O$ hospital foi selecionado devido a três características: (i) a possibilidade de acesso e disponibilidade dos funcionários para colaboração na execução da pesquisa; (ii) a existência de iniciativas de melhoria prévias, alinhadas ao objetivo desse estudo e (iii) o interesse das lideranças da organização no uso de diferentes métodos de solução de problemas.

O trabalho iniciou com a delimitação do sistema sob análise, etapa na qual foram definidos o método de solução de problemas utilizado, o processo abordado e os problemas enfatizados. Na mesma etapa foi realizada a seleção da unidade do hospital para execução do estudo, seguindo então para a aplicação do método. As etapas do estudo, que incluem a aplicação do FRAM, são representadas na figura 1.

Figura 1 - Etapas da execução do estudo

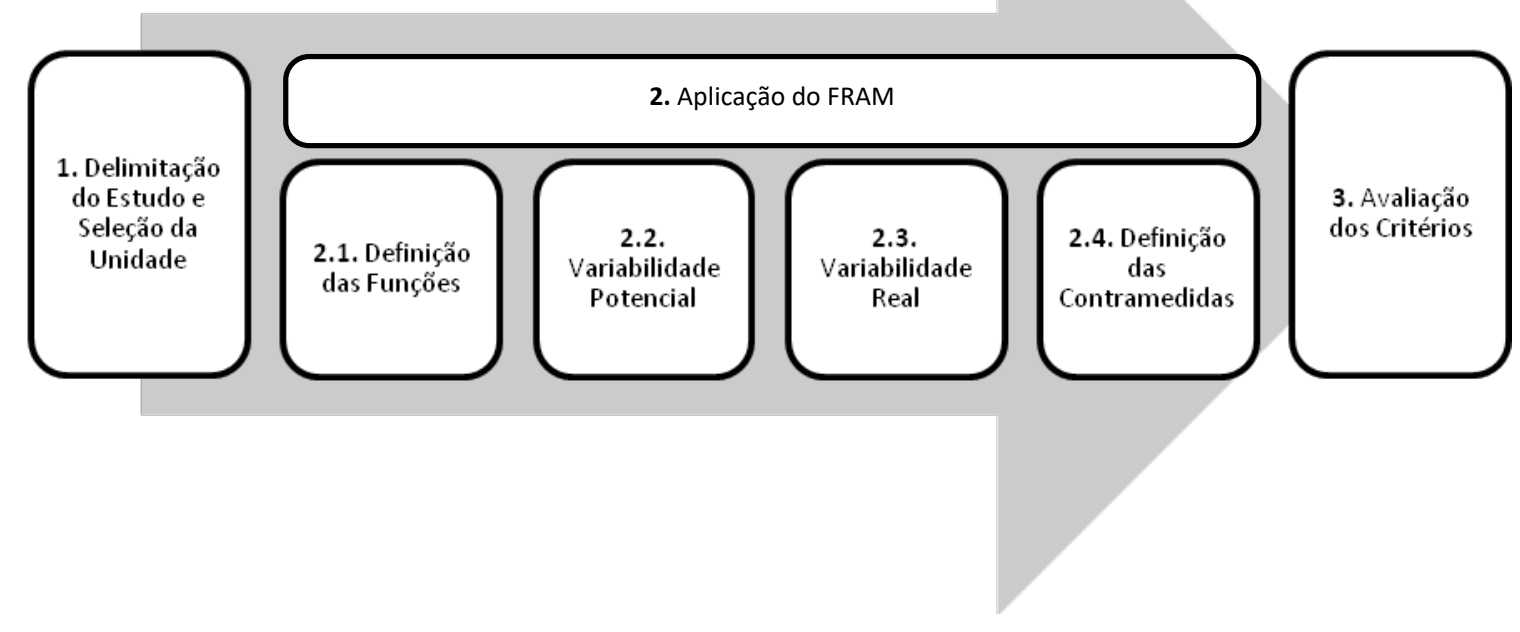

A etapa final do estudo, realizada após a conclusão da aplicação do FRAM, consiste na avaliação dos critérios selecionados para analisar os resultados do método. As contramedidas propostas foram avaliadas por três 
assessores de operações assistenciais da administração central do hospital, com auxílio de um assessor da farmácia, por possuírem conhecimento do processo e uma visão global das unidades, permitindo que classificassem as contramedidas de acordo com dois critérios: a) dificuldade de implementação e b) a possibilidade de replicação em outras unidades do hospital. A forma de avaliação desses critérios é apresentada no quadro 1. Os avaliadores não participaram de nenhuma etapa da aplicação do FRAM, tomando conhecimento das contramedidas propostas apenas no momento da avaliação. A avaliação foi realizada em conjunto pelos quatro assessores ao longo de duas reuniões, buscando consenso quanto aos valores mais adequados para cada ação.

Após a avaliação das contramedidas, os valores correspondentes às classificações nesses dois critérios foram multiplicados para gerar um indicador único denominado Prioridade, do tipo maior melhor, que permite a priorização das contramedidas para implementação. Além disso, também foram adotados critérios para a avaliação da eficiência do método em si: o tempo necessário para aplicação, o número de envolvidos necessário, a necessidade de capacitação prévia e o número total de oportunidades de melhoria identificadas.

Quadro 1 - Avaliação dos critérios, com os valores definidos e suas respectivas interpretações

Critério: Dificuldade de implementação

\begin{tabular}{|l|l|}
\hline Valor & Interpretação \\
\hline 1 & Ação de implementação muito difícil \\
\hline 3 & Ação com dificuldade média de implementação \\
\hline 9 & Ação de implementação fácil \\
\hline
\end{tabular}

Critério: Possibilidade de replicação em outras unidades

\begin{tabular}{|l|l|}
\hline Valor & Interpretação \\
\hline 1 & Ação válida apenas para unidade onde foi proposta \\
\hline 3 & Ação válida para algumas unidades \\
\hline 9 & Ação válida para todo o hospital \\
\hline
\end{tabular}

\subsection{Coleta dos dados}

A coleta dos dados necessários para construção do modelo e a aplicação do método se deram ao longo de quatro reuniões, com duração aproximada de uma hora cada, realizadas no período de duas semanas. As reuniões ocorreram durante o horário de trabalho da equipe. Dessa forma todos envolvidos foram 
convidados a colaborar nos momentos que tivessem disponibilidade, priorizando o atendimento aos pacientes. Ao todo 11 funcionários da equipe de enfermagem participaram do estudo, por indicação da liderança da equipe de enfermagem da unidade e por estarem disponíveis nos momentos das reuniões de trabalho. Todas as informações fornecidas pela equipe foram validadas pela liderança da equipe de enfermagem da unidade selecionada, de forma a garantir que refletissem a realidade da área.

Na primeira reunião o método foi apresentado à equipe da unidade, o objetivo e escopo do estudo foram expostos e iniciou-se o mapeamento das funções referente aos processos de prescrição e administração de medicamentos. Os presentes foram provocados a descrever as atividades que realizam rotineiramente, etapa por etapa, da forma como as executam. Por meio dessa descrição foram mapeadas as funções presentes no processo.

Com a lista de funções do processo definida, iniciou-se a definição dos aspectos de cada função. Os participantes foram convidados a descrever em detalhes a execução de cada uma das atividades previamente mencionadas, dando destaque para recursos necessários, controles existentes, pré-requisitos, prazos ou restrições de tempo. A equipe também foi questionada a respeito da variabilidade potencial dos resultados, comentando sobre a precisão e do tempo de execução de cada uma das funções. As informações coletadas foram então inseridas no software FRAM Model Visualiser (FMV), para construção do modelo FRAM do processo.

O modelo FRAM foi apresentado à equipe que validou a coerência com o processo real. Com base no modelo a variabilidade real foi avaliada, convidando a equipe a descrever como alterações nas atividades poderiam influenciar nas interações entre as funções, e qual seria o impacto decorrente na segurança do paciente. A etapa final foi a definição de contramedidas, nessa etapa os participantes foram incentivados a sugerir alterações que permitissem um maior controle em situações de variabilidade descontrolada, buscando formas de detectar a variabilidade e impedir impactos negativos no paciente.

Os 11 funcionários contribuíram em diferentes momentos durante a coleta de informações, dentre os quais três - dois enfermeiros e um técnico de enfermagem - acompanharam todas as etapas da aplicação do método. Os participantes do estudo não receberam capacitação prévia no FRAM, que foi 
introduzido à equipe apenas na primeira reunião de trabalho. $\mathrm{O}$ autor do presente estudo atuou como orientador para aplicação do método, buscando não influenciar em nenhum momento nas informações cedidas pelos participantes, que tiveram liberdade para opinar em todas as etapas da construção do modelo FRAM e sugeriram as contramedidas para as oportunidades identificadas. Três sessões de observações não participantes foram utilizadas para permitir um melhor entendimento do processo abordado. As quatro reuniões de trabalho durante a aplicação do método foram registradas em atas e um diário de campo foi utilizado para registro das reuniões e das observações de campo. Entrevistas de participação voluntária foram conduzidas com os participantes do trabalho para melhor entendimento das percepções a respeito do método utilizado. Foram entrevistados dois enfermeiros e um técnico de enfermagem, membros da equipe de aplicação do FRAM, os quais acompanharam todas as etapas do método. As entrevistas foram gravadas mediante autorização dos participantes. Assim, a coleta de dados envolveu observações, análise de procedimentos, reuniões e entrevistas. O quadro 2 apresenta as fontes utilizadas em cada etapa do estudo.

Quadro 2 - Fontes de dados associadas às diferentes etapas do estudo

\begin{tabular}{|c|c|c|c|c|c|}
\hline & \multirow[b]{2}{*}{ Etapa do estudo } & \multicolumn{4}{|c|}{ Fonte de dados } \\
\hline & & $\begin{array}{l}\text { Observação } \\
\text { não } \\
\text { participante }\end{array}$ & $\begin{array}{c}\text { Análise de } \\
\text { procedimentos }\end{array}$ & $\begin{array}{l}\text { Observação } \\
\text { participante }\end{array}$ & $\begin{array}{l}\text { Entrevista } \\
\text { semi- } \\
\text { estruturada }\end{array}$ \\
\hline & 1. Delimitação do estudo e & $\mathrm{x}$ & $x$ & $x$ & \\
\hline & Seleção da unidade & $\mathrm{x}$ & & $\mathrm{X}$ & \\
\hline \multirow{5}{*}{ 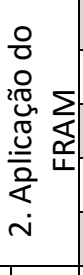 } & 2.1. Definição das funções & $\mathrm{X}$ & $\mathrm{x}$ & & \\
\hline & 2.2. Variabilidade potencial & $\mathrm{x}$ & $x$ & & \\
\hline & 2.3. Variabilidade Real & & & $x$ & \\
\hline & 2.4. Definição das Contramedidas & & & $\mathrm{X}$ & \\
\hline & 3. Avaliação dos critérios & & & $X$ & $x$ \\
\hline & Total & $6 \mathrm{~h}$ & & $12 \mathrm{~h}$ & $1 \mathrm{~h}$ \\
\hline
\end{tabular}

O estudo passou por avaliação e aprovação prévia pelo Comitê de Ética do hospital. Foi esclarecido a todos os envolvidos que os resultados seriam publicados, com sigilo garantido. Os participantes das entrevistas registraram sua concordância voluntária mediante assinatura de um termo de consentimento livre e esclarecimento. 


\subsection{Análise dos dados}

A análise dos dados foi realizada por exame do conteúdo das entrevistas, dos registros de observações e pela compilação dos resultados da avaliação das contramedidas propostas, conforme os critérios adotados. Durante a aplicação do FRAM, informações específicas foram necessárias para a conclusão de cada etapa e o início da seguinte. Os dados analisados para a obtenção dessas informações em cada etapa são apresentados no quadro 3.

Quadro 3 - Informações buscadas nas fontes de dados, guiando a análise de conteúdo

\begin{tabular}{|c|c|c|}
\hline Dado analisado & Informação buscada nas fontes de dados & $\begin{array}{c}\text { Etapa do estudo } \\
\text { relacionada }\end{array}$ \\
\hline Problemas & $\begin{array}{l}\text { Incidentes já observados anteriormente, diferenças entre } \\
\text { o processo realizado e os procedimentos, indicadores } \\
\text { abaixo de metas estipuladas. }\end{array}$ & $\begin{array}{l}\text { 1. Delimitação } \\
\text { do estudo e } \\
\text { Seleção da } \\
\text { unidade }\end{array}$ \\
\hline Funções & $\begin{array}{l}\text { Como a atividade é executada, que ajustes são } \\
\text { necessários para diferentes condições, como a atividade é } \\
\text { preparada, quais os recursos necessários, quais condições } \\
\text { devem ser cumpridas, como os resultados são } \\
\text { controlados. }\end{array}$ & $\begin{array}{l}\text { 2. Aplicação do } \\
\text { FRAM }\end{array}$ \\
\hline Variabilidade Potencial & $\begin{array}{l}\text { De que forma o resultado de cada atividade pode variar, } \\
\text { qual a precisão da atividade, qual a pontualidade da } \\
\text { entrega dos resultados, que fatores externos podem } \\
\text { influenciar no processo. }\end{array}$ & $\begin{array}{l}\text { 2. Aplicação do } \\
\text { FRAM }\end{array}$ \\
\hline Variabilidade Real & $\begin{array}{l}\text { Como um ajuste em determinada atividade impactará nas } \\
\text { demais funções do processo, possibilidade do impacto } \\
\text { interferir na segurança do paciente. }\end{array}$ & $\begin{array}{l}\text { 2. Aplicação do } \\
\text { FRAM }\end{array}$ \\
\hline Contramedidas & $\begin{array}{l}\text { Maneiras de impedir que ajustes realizados aos longo das } \\
\text { atividades impactem na segurança do paciente, como } \\
\text { detectar e controlar a variabilidade nas atividades críticas. }\end{array}$ & $\begin{array}{l}\text { 2. Aplicação do } \\
\text { FRAM }\end{array}$ \\
\hline Critérios avaliados & $\begin{array}{l}\text { Experiência necessária para aplicação do método, } \\
\text { dificuldades relatadas pelos participantes, pontos } \\
\text { positivos observados pelos envolvidos, dificuldade de } \\
\text { implementação e possibilidade de replicação das } \\
\text { contramedidas, tempo necessário para a aplicação do } \\
\text { método, número de envolvidos. }\end{array}$ & $\begin{array}{l}\text { 3. Avaliação dos } \\
\text { critérios }\end{array}$ \\
\hline
\end{tabular}

\subsection{Apresentação do processo}

O trabalho foi aplicado em uma unidade de internação do hospital universitário. A unidade conta com dois dispensários eletrônicos para disponibilização dos medicamentos, sendo abastecidos pelos profissionais da farmácia central do hospital. Além dos profissionais de enfermagem, a unidade 
é frequentada por médicos e outros profissionais dos diferentes setores que atendem às necessidades dos pacientes internados.

O processo de prescrição e administração de medicamentos na unidade inicia pela avaliação do paciente pelo médico, na admissão do paciente. Após a avaliação, o médico define os medicamentos a serem prescritos e insere a prescrição no sistema de prescrição eletrônica do hospital. A prescrição eletrônica fica então disponível no sistema para o aprazamento. O processo de aprazamento é realizado pelos técnicos de enfermagem que, ao receber a prescrição médica, definem os horários de administração obedecendo à periodicidade determinada pelo médico, realizando alterações nos horários conforme necessário, ou contatando o enfermeiro responsável caso sejam necessárias alterações nos medicamentos.

Após o aprazamento eletrônico, o técnico responsável por essa atividade imprime duas vias da prescrição, uma destinada à pasta do paciente - que fica permanentemente alocada no posto de enfermagem da unidade, para consulta de profissionais de todas as áreas - e uma segunda via, denominada contracheque, destinada ao uso da equipe de enfermagem e entregue pessoalmente ao técnico de enfermagem responsável pelo paciente. Ao receber o contracheque, o técnico de enfermagem verifica os medicamentos prescritos e separa etiquetas adesivas que contém o nome do paciente, destinadas à identificação dos medicamentos, nas quais preenche o nome do medicamento prescrito, a dose, a via de administração, a data e a hora. Cada técnico de enfermagem é responsável pelo atendimento de até seis pacientes, para os quais efetua todas as atividades de preparo dos medicamentos.

Todo o preparo das etiquetas é realizado no posto de enfermagem, de acordo com as informações do contracheque. Ao concluir essa tarefa, os técnicos se deslocam até a sala onde estão localizados os dispensários eletrônicos, para a retirada dos medicamentos, onde permanecem até retirar os medicamentos de todos os pacientes pelos quais são responsáveis. O dispensário eletrônico permite a retirada dos medicamentos de acordo com o aprazamento da prescrição eletrônica, liberando os medicamentos prescritos entre duas horas antes e uma hora depois do horário registrado na prescrição.

Para alguns medicamentos é necessário realizar diluição. Essa atividade demanda que o técnico de enfermagem dilua em soro fisiológico o medicamento 
disponível no dispensário, até a dose prescrita. A diluição deve ser realizada de acordo com os procedimentos registrados no livro de diluições, disponível no posto de enfermagem, que orientam como calcular a dose necessária para atender à prescrição. Cada técnico deve também preparar salinizações, que serão utilizadas para higienização do acesso dos pacientes em caso de medicamentos intravenosos. Os medicamentos e as diluições são então rotulados com as etiquetas previamente preparadas, e então conferidos de acordo com o contracheque. $O$ técnico de enfermagem então se desloca até 0 leito de seus pacientes para administrar o medicamento.

Ao localizar o paciente, cabe ao profissional realizar a conferência na beira do leito, verificando a etiqueta de cada medicamento com a pulseira do paciente, que contém seu nome, antes da administração do conteúdo. Medicamentos que necessitam acesso para administração, exigem preparo do acesso, que deve ser previamente verificado e higienizado com solução salina pelo técnico de enfermagem, para segurança do paciente. Após a medicação de todos pacientes pelos quais é responsável, o técnico de enfermagem registra nos contracheques os medicamentos administrados e o horário, posteriormente inserindo essas informações na prescrição eletrônica. Finalizando o processo, os utensílios de uso único são então descartados e o técnico realiza a higiene dos demais utensílios.

\section{RESULTADOS E DISCUSSÃO}

\subsection{Modelo FRAM}

Foram identificadas 18 funções no processo de prescrição e medicação da unidade. Três dessas funções são realizadas pelo corpo médico, referentes ao processo de prescrição, sendo que as demais estão sob responsabilidade dos técnicos de enfermagem. O modelo gerado no FMV pode ser visualizado na figura 2. As funções representadas em azul no modelo FRAM são executadas por médicos, as demais, em branco, são funções executadas pelos técnicos de enfermagem. Funções de maior variabilidade potencial são sinalizadas por uma onda senoidal na representação do modelo. No quadro 4 são listadas as funções 
que compõem o modelo FRAM, o número de acoplamentos delas, suas saídas e a variabilidade potencial dessas. 
Figura 2 - Modelo FRAM do processo de prescrição e administração de medicamentos

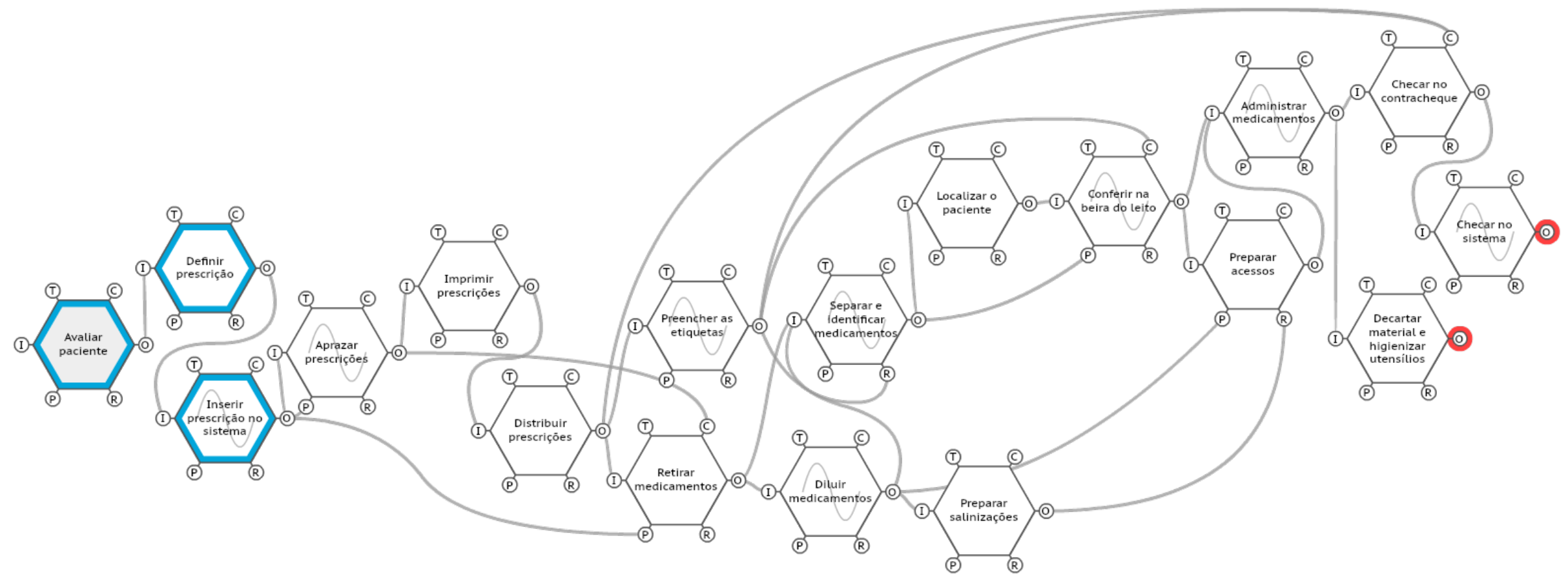


Quadro 4 - Funções do modelo, número de acoplamentos a montante (M) e a jusante (J), saídas e variabilidade potencial.

\begin{tabular}{|c|c|c|c|}
\hline Função & Acoplamentos & Saída & Variabilidade Potencial da Saída \\
\hline Avaliar paciente & $1(\mathrm{~J})$ & Paciente avaliado & $\begin{array}{l}\text { A tempo } \\
\text { Preciso }\end{array}$ \\
\hline Definir prescrição & $1(\mathrm{M})+1(\mathrm{~J})$ & $\begin{array}{l}\text { Prescrição } \\
\text { definida }\end{array}$ & $\begin{array}{l}\text { A tempo } \\
\text { Preciso }\end{array}$ \\
\hline $\begin{array}{l}\text { Inserir prescrição } \\
\text { no sistema }\end{array}$ & $1(\mathrm{M})+3(\mathrm{~J})$ & $\begin{array}{l}\text { Prescrição } \\
\text { eletrônica } \\
\text { Prescrição vigente }\end{array}$ & $\begin{array}{l}\text { Muito tarde: A prescrição deve ser inserida } \\
\text { no sistema até o horário limite (19h), ou o } \\
\text { paciente é considerado sem prescrição } \\
\text { vigente } \\
\text { Aceitável }\end{array}$ \\
\hline Aprazar prescrições & $2(\mathrm{M})+2(\mathrm{~J})$ & $\begin{array}{l}\text { Prescrição } \\
\text { aprazada }\end{array}$ & $\begin{array}{l}\text { A tempo } \\
\text { Aceitável: A permanência de } \\
\text { medicamentos prescritos como 'se } \\
\text { necessário' pode causar erros. }\end{array}$ \\
\hline $\begin{array}{l}\text { Imprimir } \\
\text { prescrições }\end{array}$ & $1(\mathrm{M})+1(\mathrm{~J})$ & $\begin{array}{l}\text { Prescrição } \\
\text { impressa }\end{array}$ & $\begin{array}{l}\text { A tempo } \\
\text { Preciso }\end{array}$ \\
\hline $\begin{array}{l}\text { Distribuir } \\
\text { prescrições }\end{array}$ & $1(\mathrm{M})+3(\mathrm{~J})$ & Contracheque & $\begin{array}{l}\text { A tempo } \\
\text { Preciso }\end{array}$ \\
\hline $\begin{array}{l}\text { Preencher as } \\
\text { etiquetas }\end{array}$ & $1(\mathrm{M})+3(\mathrm{~J})$ & $\begin{array}{l}\text { Etiquetas } \\
\text { preenchidas }\end{array}$ & $\begin{array}{l}\text { A tempo } \\
\text { Impreciso: Nem toda etiqueta é } \\
\text { preenchida, para acelerar o processo. }\end{array}$ \\
\hline $\begin{array}{l}\text { Retirar } \\
\text { medicamentos }\end{array}$ & $3(\mathrm{M})+2(\mathrm{~J})$ & $\begin{array}{l}\text { Medicamentos } \\
\text { Medicamentos em } \\
\text { pó }\end{array}$ & $\begin{array}{l}\text { A tempo } \\
\text { Preciso }\end{array}$ \\
\hline $\begin{array}{l}\text { Diluir } \\
\text { medicamentos }\end{array}$ & $1(\mathrm{M})+3(\mathrm{~J})$ & $\begin{array}{l}\text { Medicamentos } \\
\text { diluídos }\end{array}$ & $\begin{array}{l}\text { A tempo } \\
\text { Aceitável: Para penicilina pode ocorrer } \\
\text { erros }\end{array}$ \\
\hline $\begin{array}{l}\text { Separar e } \\
\text { identificar } \\
\text { medicamentos }\end{array}$ & $3(\mathrm{M})+2(\mathrm{~J})$ & $\begin{array}{l}\text { Medicamentos } \\
\text { preparados }\end{array}$ & $\begin{array}{l}\text { A tempo } \\
\text { Aceitável: Ocorrem interrupções } \\
\text { constantes durante a execução dessa } \\
\text { atividade }\end{array}$ \\
\hline $\begin{array}{l}\text { Preparar } \\
\text { salinizações }\end{array}$ & $1(\mathrm{M})+1(\mathrm{~J})$ & Salinizações & $\begin{array}{l}\text { A tempo } \\
\text { Preciso }\end{array}$ \\
\hline Localizar o paciente & $1(\mathrm{M})+1(\mathrm{~J})$ & $\begin{array}{l}\text { Paciente } \\
\text { localizado }\end{array}$ & $\begin{array}{l}\text { A tempo } \\
\text { Preciso }\end{array}$ \\
\hline $\begin{array}{l}\text { Conferir na beira do } \\
\text { leito }\end{array}$ & $3(M)+2(J)$ & $\begin{array}{l}\text { Medicamentos } \\
\text { checados }\end{array}$ & $\begin{array}{l}\text { A tempo } \\
\text { Aceitável }\end{array}$ \\
\hline Preparar acessos & $3(\mathrm{M})+1(\mathrm{~J})$ & $\begin{array}{l}\text { Acesso } \\
\text { higienizado }\end{array}$ & $\begin{array}{l}\text { A tempo } \\
\text { Preciso }\end{array}$ \\
\hline $\begin{array}{l}\text { Administrar } \\
\text { medicamentos }\end{array}$ & $2(\mathrm{M})+2(\mathrm{~J})$ & $\begin{array}{l}\text { Medicamentos } \\
\text { administrados }\end{array}$ & $\begin{array}{l}\text { A tempo } \\
\text { Aceitável: Paciente pode se recusar a } \\
\text { receber o medicamento }\end{array}$ \\
\hline $\begin{array}{l}\text { Checar no } \\
\text { contracheque }\end{array}$ & $3(\mathrm{M})+1(\mathrm{~J})$ & $\begin{array}{l}\text { Contracheque } \\
\text { checado }\end{array}$ & $\begin{array}{l}\text { A tempo } \\
\text { Preciso }\end{array}$ \\
\hline Checar no sistema & $1(\mathrm{M})$ & $\begin{array}{l}\text { Prescrição } \\
\text { atualizada }\end{array}$ & $\begin{array}{l}\text { Muito tarde: Comumente é necessário } \\
\text { aguardar por computador disponível. } \\
\text { Aceitável }\end{array}$ \\
\hline $\begin{array}{l}\text { Descartar material } \\
\text { e higienizar } \\
\text { utensilios }\end{array}$ & $1(\mathrm{M})$ & $\begin{array}{l}\text { Material } \\
\text { higienizado }\end{array}$ & $\begin{array}{l}\text { A tempo } \\
\text { Preciso }\end{array}$ \\
\hline
\end{tabular}

O modelo FRAM permite visualizar a interação das funções necessárias para execução do processo, demonstrando como estão acopladas e quais as Revista Produção Online. Florianópolis, SC, v. 19, n. 1, p. 102-128, 2019. 
potenciais fontes de maior variabilidade. Essa visualização permite instanciações do impacto da variabilidade ao longo do processo, possibilitando determinar quais funções serão possivelmente afetadas em determinada situação. Um exemplo é a função 'preencher as etiquetas', a qual corresponde à tarefa na qual o técnico de enfermagem preenche as etiquetas adesivas com as informações dos medicamentos a serem administrados. Essa função é disparada pelo recebimento do contracheque, saída da função 'distribuir prescrições', e as etiquetas a serem preenchidas são o recurso necessário para sua execução. O controle dessa função é o procedimento Seis Certos, um conjunto de boas práticas adotado pelos profissionais de enfermagem como forma de minimizar erros de medicação. A função 'preencher as etiquetas' foi considerada imprecisa, pois, de acordo com os relatos dos funcionários, o preenchimento das etiquetas nem sempre é completo. Acopladas a jusante de 'preencher as etiquetas' estão as funções 'separar e identificar medicamentos', 'conferir na beira do leito' e 'checar no contracheque'; as quais podem ser impactadas caso o preenchimento das etiquetas esteja inadequado. Identificada essa fonte de variabilidade na função 'preencher as etiquetas', a equipe foi convidada a propor contramedidas para o controle dessa variabilidade. Dessa forma, a equipe propôs a ação: Etiquetas virem prontas do dispensário, ou de uma impressora paralela e conectada a ele, sendo disponibilizadas junto com cada medicamento; essa ação reduziria o potencial de erro humano e liberaria mais tempo para os técnicos de enfermagem desempenharem com maior tranquilidade a função adjacente 'separar e identificar medicamentos'.

\subsection{Contramedidas propostas e priorização}

A aplicação do FRAM resultou em 18 contramedidas propostas, as quais foram classificadas com uma média de 3,2 para o critério dificuldade de implementação e 7,9 para possibilidade de replicação da ação entre as unidades do hospital. A média do indicador Prioridade é 27,2, diante um máximo possível de 81. As ações de melhoria propostas, bem como suas avaliações, são apresentadas no quadro 5. 
Quadro 5 - Contramedidas pontuadas nos critérios de avaliação

\begin{tabular}{|c|c|c|c|c|}
\hline $\mathbf{N}^{\circ}$ & Contramedida & Dificuldade & Replicação & Prioridade \\
\hline 1 & $\begin{array}{l}\text { Etiquetas virem prontas do dispensário/impressora } \\
\text { paralela, sendo disponibilizadas junto com cada } \\
\text { medicamento. }\end{array}$ & 1 & 9 & 9 \\
\hline 2 & $\begin{array}{l}\text { Etiquetas impressas, que necessitem preenchimento } \\
\text { manual, serem maiores }\end{array}$ & 1 & 9 & 9 \\
\hline 3 & $\begin{array}{l}\text { Isolar o técnico de enfermagem durante a atividade de } \\
\text { preparo do medicamento }\end{array}$ & 3 & 9 & 27 \\
\hline 4 & $\begin{array}{l}\text { Pessoa dedicada a atendimento de demandas nos horários } \\
\text { de pico }\end{array}$ & 1 & 9 & 9 \\
\hline 5 & $\begin{array}{l}\text { Bancada de preparo de medicamentos feita de material } \\
\text { que facilite a higiene }\end{array}$ & 3 & 9 & 27 \\
\hline 6 & $\begin{array}{l}\text { Profissional da farmácia para o preparo de salinizações, de } \\
\text { acordo com a necessidade de pacientes }\end{array}$ & 3 & 9 & 27 \\
\hline 7 & $\begin{array}{l}\text { Permitir que os técnicos de enfermagem realizem a punção } \\
\text { venosa sem a presença de um profissional de enfermagem }\end{array}$ & 1 & 1 & 1 \\
\hline 8 & $\begin{array}{l}\text { Leitor de código de barras para checagem na beira do leito } \\
\text { (checagem eletrônica) }\end{array}$ & 1 & 9 & 9 \\
\hline 9 & $\begin{array}{l}\text { Regras de uso para utilização dos computadores das } \\
\text { unidades e iniciativa de conscientização quanto às regras }\end{array}$ & 9 & 9 & 81 \\
\hline 10 & $\begin{array}{l}\text { Computadores nos quartos, permitindo atualizações na } \\
\text { prescrição em tempo real }\end{array}$ & 1 & 9 & 9 \\
\hline 11 & $\begin{array}{l}\text { Reorganizar sala com os dispensários para centralizar no } \\
\text { mesmo local o preparo de medicamentos e alguns } \\
\text { computadores }\end{array}$ & 1 & 9 & 9 \\
\hline 12 & $\begin{array}{l}\text { Iniciativa de educação para o uso do apoio do Centro de } \\
\text { Informação de Medicamentos (CIM) em caso de dúvidas } \\
\text { durante as diluições }\end{array}$ & 9 & 9 & 81 \\
\hline 13 & $\begin{array}{l}\text { Telefone no posto de enfermagem/local de preparo dos } \\
\text { medicamentos, dedicado exclusivamente para ligações } \\
\text { para o CIM }\end{array}$ & 9 & 9 & 81 \\
\hline 14 & Diluições serem realizadas na farmácia & 1 & 9 & 9 \\
\hline 15 & Aprazamento inicial feito pelo farmacêutico & 1 & 9 & 9 \\
\hline 16 & $\begin{array}{l}\text { Dispensário eletrônico demonstrar o número de } \\
\text { comprimidos necessários de acordo com a dosagem do } \\
\text { medicamento disponível }\end{array}$ & 1 & 3 & 3 \\
\hline 17 & Bandeja maior, com separadores fixos & 9 & 9 & 81 \\
\hline 18 & $\begin{array}{l}\text { Carrinho para medicamentos por leito, com espaço para } \\
\text { descarte }\end{array}$ & 3 & 3 & 9 \\
\hline
\end{tabular}

Avaliando-se as médias de cada critério com base na régua adotada para interpretação dos valores, verifica-se que as ações de melhoria resultantes do trabalho foram consideradas, em média, de dificuldade intermediária para aplicação. Visualizando individualmente as contramedidas, verifica-se que 10 foram pontuadas como de implementação muito difícil, quatro de dificuldade média e quatro de fácil implementação, indicando que a maioria das contramedidas propostas possuem obstáculos para serem implementadas. Em relação à possibilidade de replicação, o valor médio da pontuação indica que as 
contramedidas propostas são válidas para a maior parte do hospital; o que é confirmado ao se verificar a avaliação individual, na qual 15 das 18 ações propostas foram pontuadas como válidas para todo o hospital. O indicador Prioridade sinaliza quatro ações com pontuação máxima nesse critério, as quais seriam as ações recomendadas para iniciar a implementação das contramedidas, por possuírem maior abrangência e menor dificuldade de implementação. Como exemplo das ações de maior prioridade, podem ser citadas as contramedidas 'iniciativa de educação para o uso do apoio do Centro de Informação de Medicamentos (CIM) em caso de dúvidas durante as diluições' e 'telefone no posto de enfermagem/local de preparo dos medicamentos, dedicado exclusivamente para ligações para o CIM'. Essas são ações complementares, que visam reduzir a possibilidade de erros no desempenho da função 'diluir medicamentos', cuja precisão é considerada apenas aceitável. O CIM é um recurso existente na instituição, porém, segundo a equipe, pouco utilizado, por isso a necessidade da ação de educação para o incentivo do uso. Um telefone dedicado para contato com o CIM, localizado no ambiente de preparo dos medicamentos, facilitaria e incentivaria o uso desse recurso, sem necessidade de investimentos significativos e com possibilidade de replicação para todas as unidades do hospital, justificando a maior prioridade dessas ações.

\subsection{Avaliação do uso do FRAM}

A grande abrangência das ações propostas pode ser justificada pela natureza da unidade onde o estudo foi realizado, a qual mantém diversas similaridades com as demais unidades de internação do hospital, além de atender pacientes de diversas especialidades clínicas, sendo frequentada por profissionais de diferentes áreas, permitindo à equipe da unidade conviver com práticas de outras partes da instituição. O número elevado de ações pontuadas como de implementação muito difícil pode ser reflexo da necessidade de investimentos elevados para algumas dessas ações, o que não foi considerado pela equipe ao sugerir as contramedidas. Um exemplo é a contramedida 'Computadores nos quartos, permitindo atualizações na prescrição em tempo real', para qual a dificuldade de implantação reflete a necessidade de investimento para a aquisição de computadores para todos os quartos, algo 
inviável diante do orçamento atual da instituição, segundo os responsáveis pela avaliação da ação. Também impacta na dificuldade o fato das melhorias, mesmo que relativas à atuação de profissionais de outras áreas relecionadas com o processo, terem sido propostas apenas por membros da equipe de enfermagem, como ocorre com a ação que propõe um profissional da farmácia para o preparo das salinizações.

Um resumo dos resultados obtidos, de acordo com os critérios adotados, é apresentado no quadro 6.

\begin{tabular}{|c|c|}
\hline Critério de avaliação & Resultados FRAM \\
\hline Número de ações de contramedidas propostas & 18 \\
\hline Dificuldade de implementação das ações (média) & 3,2 \\
\hline Abrangência das ações (média) & 7,9 \\
\hline Tempo necessário para aplicação & $4 h$ \\
\hline Número de envolvidos na equipe & 11 \\
\hline Necessidade de capacitação da equipe & Sem capacitação prévia \\
\hline
\end{tabular}

Nenhum dos participantes entrevistados afirmou ter conhecimento prévio do FRAM, ainda assim foi unânime a percepção de que o método não é de difícil aplicação ou compreensão, sendo considerado didático por um dos respondentes. Um dos membros da equipe alegou que as informações necessárias para aplicar o método são bem evidentes em seu dia-a-dia; relato esse que reflete uma das premissas utilizadas no desenvolvimento do FRAM, de ser um método que focasse na natureza das atividades rotineiras (Hollnagel, 2012). Os membros da equipe não relataram dificuldades relacionadas ao método, porém dois dos entrevistados citaram a dificuldade de participar da aplicação do FRAM durante o expediente, no próprio ambiente de trabalho. Esse relato justifica-se pelo fato dos profissionais não terem se desvencilhado do atendimento aos pacientes durante a participação no estudo, incorrendo em constantes interrupções para execução de outras tarefas. Essa mesma condição reflete na percepção sobre a adequação do tempo dedicado ao desenvolvimento do trabalho. Apesar de ter a duração considerada adequada por todos os consultados, um dos respondentes sugeriu que haveria melhor aproveitamento se houvesse dedicação exclusiva nesse período. O local onde as reuniões de trabalho foram conduzidas, o posto de enfermagem da unidade, também foi 
citado por facilitar interrupções, todavia essa condição corresponde à rotina de trabalho da equipe, refletindo inclusive nas contramedidas propostas, as quais incluem duas ações de melhoria que objetivam reduzir as interrupções durante o preparo de medicamentos. Hollnagel et al. (2014) recomendam que a aplicação do método seja conduzida no ambiente de trabalho dos participantes, de forma a incorporar a sensação do ambiente.

Entre os pontos positivos relatados, destaca-se a análise aprofundada do trabalho rotineiro, que além de proporcionar uma visualização didática do processo, pode servir para identificar discrepâncias entre as maneiras que diferentes funcionários executam a mesma tarefa, conforme alegou um dos participantes. Também foi citado como um ponto positivo o aspecto da busca por melhorias inerente ao método. Os entrevistados foram unânimes ao afirmar que não acreditam na possibilidade de encontrar os mesmos resultados do trabalho sem a utilização de um método estruturado, bem como todos demonstraram interesse na aplicação do FRAM em outros processos e setores do hospital. Resultados semelhantes são observados no trabalho conduzido por Sujan e Felici (2012), no qual os participantes alegaram que o FRAM proporcionou uma melhor compreensão de como funcionários lidam com a variabilidade e como isso afeta o sistema.

\section{CONCLUSÕES}

Sistemas sócio-técnicos complexos como o ambiente hospitalar possuem particularidades que exigem uma abordagem robusta para solução dos seus problemas. O presente trabalho avaliou o desempenho do FRAM, um método relativamente novo, nesse contexto. Para esse fim, as ações de melhoria propostas como resultado da aplicação do FRAM foram classificadas de acordo com os critérios de dificuldade de implementação e a possibilidade de replicação entre as unidades do hospital, sendo os valores dos dois critérios multiplicados para gerar um indicador denominado Prioridade, utilizado para priorizar a implementação das contramedidas. Também foram avaliados 0 tempo necessário para aplicação do método, o número de envolvidos, a necessidade de capacitação e o número total de contramedidas identificadas. 
O FRAM proporcionou aos participantes do estudo uma visão didática do processo avaliado, facilitando identificar divergências entre o trabalho como realizado e o trabalho como imaginado. Destaca-se a facilidade de aplicação do método, que exige basicamente uma boa compreensão de como o trabalho é executado, não demandando mais tempo do que o aceitável ou uma quantidade inviável de envolvidos. Os participantes do estudo atribuíram as oportunidades de melhorias identificadas ao uso do FRAM, e alegam ver possibilidade de aplicação do método em outras unidades e processos do hospital, demonstrando satisfação com os resultados alcançados.

\section{REFERÊNCIAS}

ALM, Helen; WOLTJER, Rogier. Patient safety investigation through the lens of FRAM. Human factors: a system view of human, technology and organisation. Maastricht, The Netherlands: Shaker Publishing, p. 153-65, 2010.

BATES, David W. Using information technology to reduce rates of medication errors in hospitals. BMJ: British Medical Journal, v. 320, n. 7237, p. 788, 2000. https://doi.org/10.1136/bmi.320.7237.788

BATES, David W. et al. Relationship between medication errors and adverse drug events. Journal of general internal medicine, v. 10, n. 4, p. 199-205, 1995.

BELMONTE, Fabien et al. Interdisciplinary safety analysis of complex sociotechnological systems based on the functional resonance accident model: An application to railway trafficsupervision. Reliability Engineering \& System Safety, v. 96, n. 2, p. 237-249, 2011.

BRAITHWAITE, Jeffrey; RUNCIMAN, William B.; MERRY, Alan F. Towards safer, better healthcare: harnessing the natural properties of complex sociotechnical systems. Quality and Safety in Health Care, v. 18, n. 1, p. 37-41, 2009. https://doi.org/10.1136/qshc.2007.023317

CASSIANI, Silvia Helena de Bortoli; FREIRE, Cláudia Câmara; GIMENES, Fernanda Raphael Escobar. A prescrição médica eletrônica em um hospital universitário: falhas de redação e opiniões de usuários. Revista da Escola de Enfermagem da USP, v. 37, n. 4, p. 51-60, 2003. https://doi.org/10.1590/S0080-62342003000400006

CILLIERS, PAUL. 'Complexity and postmodernism. Understanding complex systems' Reply to David Spurrett. South African Journal of Philosophy, v. 18, n. 2, p. 275278, 1999. https://doi.org/10.1080/02580136.1999.10878187

HERRERA, Ivonne A.; WOLTJER, Rogier. Comparing a multi-linear (STEP) and systemic (FRAM) method for accident analysis. Reliability Engineering \& System Safety, v. 95, n. 12, p. 1269-1275, 2010. https://doi.org/10.1016/j.ress.2010.06.003 
HOLLNAGEL, Erik. The changing nature of risk. Ergonomics Australia Journal, v. 22, n. 1-2, p. 33-46, 2008.

HOLLNAGEL, Erik. FRAM, the functional resonance analysis method: modelling complex socio-technical systems. Ashgate Publishing, Ltd., 2012.

HOLLNAGEL, Erik; GOTEMAN, Orjan. The functional resonance accident model. Proceedings of cognitive system engineering in process plant, v. 2004, p. 155161, 2004.

HOLLNAGEL, Erik; SPEZIALI, Josephine. Study on developments in accident investigation methods: a survey of the" state-of-the-art. 2008.

HOLLNAGEL, Erik; WOODS, David D.; LEVESON, Nancy. Resilience engineering: concepts and precepts. Ashgate Publishing, Ltd., 2006.

HOUNSGAARD, Jeanette. Patient safety in everyday work learning from things that go right. Center For Kvalitet, 2016.

LEVENSON, Nancy G. System safety and computers. Addison Wesley, 1995.

LEVESON, Nancy G. Evaluating Accident models using recent aerospace accidents, Part 1: Event-Based Models. 2001.

LEVESON, Nancy G. System safety engineering: back to the future. Massachusetts Institute of Technology, 2002.

LUNDBLAD, Karin et al. FRAM as a risk assessment method for nuclear fuel transportation. In: Proceedings of the 4th International Conference Working on Safety. 2008. p. 223-1.

MAGRABI, Farah et al. Using an accident model to design safe electronic medication management systems. In: Medinfo 2007: Proceedings of the 12th World Congress on Health (Medical) Informatics; Building Sustainable Health Systems. IOS Press, 2007. p. 948.

MARAIS, KAREN et al. Beyond normal accidents and high reliability organizations: The need for an alternative approach to safety in complex systems. In: Engineering Systems Division Symposium. 2004. p. 1-16.

PASIN, Simone S. Análise do preparo e administração de medicamentos: priorização dos problemas e planejamento de melhorias na perspectiva lean. Artigo (Especialização em Gestão da Produção) - UFRGS- Universidade Federal do Rio Grande do Sul, Brasil, 2015.

PATRIARCA, Riccardo; DI GRAVIO, Giulio; COSTANTINO, Francesco. A Monte Carlo evolution of the Functional Resonance Analysis Method (FRAM) to assess performance variability in complex systems. Safety science, v. 91, p. 49-60, 2017. https://doi.org/10.1016/j.ssci.2016.07.016

PICKUP, Laura et al. The dichotomy of the application of a systems approach in UK healthcare the challenges and priorities for implementation. Ergonomics, v. 61, n. 1, p. 15-25, 2018. 
RABEN, Ditte Caroline et al. Learn from what goes right: A demonstration of a new systematic method for identification of leading indicators in healthcare. Reliability Engineering \& System Safety, v. 169, p. 187-198, 2018.

ROSA, Lucio Villarinho; HADDAD, Assed Naked; DE CARVALHO, Paulo Victor Rodrigues. Assessing risk in sustainable construction using the Functional Resonance Analysis Method (FRAM). Cognition, Technology \& Work, v. 17, n. 4, p. 559-573, 2015. https://doi.org/10.1007/s10111-015-0337-z

ROSS, Al et al. A systems approach using the functional resonance analysis method to support fluoride varnish application for children attending general dental practice. Applied Ergonomics, v. 68, p. 294-303, 2018.

SAURIN, Tarcisio Abreu; ROSSO, Caroline Brum; COLLIGAN, Lacey. Towards a resilient and lean health care. Resilient Health Care III: Reconciling Work-AsImagined and Work-As-Done. UK: Ashgate, 2015.

SAWARAGI, Tetsuo; HORIGUCHI, Yukio; HINA, Akihiro. Safety analysis of systemic accidents triggered by performance deviation. In: SICE-ICASE, 2006. International Joint Conference. IEEE, 2006. p. 1778-1781.

SHOJANIA, Kaveh G. et al. Making health care safer: a critical analysis of patient safety practices. Evid Rep Technol Assess (Summ), v. 43, n. 1, p. 668, 2001.

STEEN, Riana; AVEN, Terje. A risk perspective suitable for resilience engineering.

Safety science, v. 49, n. 2, p. 292-297, 2011. https://doi.org/10.1016/j.ssci.2010.09.003

SUJAN, Mark-Alexander; FELICI, Massimo. Combining failure mode and functional resonance analyses in healthcare settings. Computer safety, reliability, and security, p. 364-375, 2012.

TEIXEIRA, Thalyta CARDOSO Alux; CASSIANI, Silvia Helena de Bortoli. Análise de causa raiz: avaliação de erros de medicação em um hospital universitário. Revista da Escola de Enfermagem da USP, v. 44, n. 1, 2010.

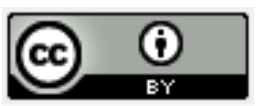

Artigo recebido em: 06/12/2017 e aceito para publicação em: 04/02/2018 DOI: http://dx.doi.org/10.14488/1676-1901.v19i1.3093 